\title{
Dopamine in the songbird auditory cortex shapes auditory preference
}

Helena J. Barr ${ }^{1,2}$, Erin M. Wall ${ }^{1,2}$, and Sarah C. Woolley ${ }^{1,2,3^{*}}$

${ }^{1}$ Integrated Program in Neuroscience, McGill University, Montreal, QC, Canada

${ }^{2}$ Center for Research on Brain, Language, and Music, McGill University, Montreal QC, Canada

${ }^{3}$ Department of Biology, McGill University, Montreal, QC, Canada

*Correspondence to sarah.woolley@mcgill.ca

keywords: auditory pallium, caudomedial nidopallium, female preference, zebra finch

Running Title: dopamine shapes song preference 


\section{ABSTRACT \\ 1}

2 In vocal communication, vocal signals can provide listeners with information and

3 also elicit motivated responses. Auditory cortical and mesolimbic reward circuits

4 are often considered to have distinct roles in these processes, with auditory

5 cortical circuits responsible for detecting and discriminating sounds and

6 mesolimbic circuits ascribing salience and modulating preference for those

7 sounds. Here, we investigated whether dopamine within auditory cortical circuits

8 themselves can shape the incentive salience of a vocal signal. Using female

9 zebra finches, who show natural preferences for vocal signals produced by males

10 ('songs'), we found that pairing passive song playback with pharmacological

11 manipulations of dopamine in the secondary auditory cortex drives changes to

12 song preferences. Plasticity of song preferences by dopamine lasted for at least

13 one week and was not influenced by norepinephrine manipulations. These data

14 suggest that dopamine acting directly in sensory processing areas can shape the

15 incentive salience of communication signals. 
INTRODUCTION

18 Vocal signals are critical for survival and reproduction in a range of species.

19 Receivers can extract substantial information from vocal signals about the

20 identity, species, or motivation of the signaller and make mate-choice and other

21 social decisions based on the incentive salience of the signal. However, there is

22 growing consensus that receivers, and their auditory systems, are not passive

23 filters, but rather they dynamically encode acoustic stimuli. ${ }^{1,2}$ Consequently, a

24 signal's salience may not be an inherent component of the signal, but instead be

25 determined by the individual receiver's internal state and experience ${ }^{3,4}$. For

26 example, in fish, frogs, and birds, reproductive status, acting through changes in

27 steroid hormones and neuromodulators, can influence auditory responses and

28 the processing of mating calls ${ }^{5-7}$. Similarly, maternal experience and reproductive

29 status dramatically shape the way that female rodents respond to pup calls, in

30 part due to neuromodulatory shaping of auditory responses ${ }^{8-10}$. Thus, the

31 response to vocal communication signals depends not only on the signal itself,

32 but also on the ascribed salience of those signals to an individual receiver.

34 Dopamine (DA) is a key modulator for ascribing incentive salience to stimuli,

35 providing the brain with information on which sensory stimuli are relevant or

36 important ${ }^{11-13}$. Dopamine neurons in the ventral tegmental area (VTA) respond to

37 reward-related stimuli in learning tasks across sensory domains ${ }^{14,15}$. Moreover,

38 dopaminergic projections from the VTA to regions like the nucleus accumbens 
39 have been found to influence a wide range of motivated behaviors ${ }^{13,14,16}$. For

40 example, DA acting within the nucleus accumbens can shape behavioral

41 responses and preferences for particular stimuli, including social stimuli. In male

42 prairie voles, injections of DA agonists into the nucleus accumbens induces

43 partner preference ${ }^{17,18}$. Thus, dopaminergic activity can serve to modulate

44 behavioral responses to communication signals.

46 However, little is known about how DA can act on areas outside the traditional

47 mesolimbic pathway to influence incentive salience for sensory stimuli. Recent

48 studies have documented that DA signals from the VTA can shape activity and

49 tuning in the auditory cortex ${ }^{19,20}$ while DA signals in the nucleus accumbens

50 relate to reward value or incentive salience ${ }^{12}$. This has led to the model that DA

51 from the VTA simultaneously acts at the level of the nucleus accumbens to shape

52 preferences and at the level of sensory cortex to correspondingly shape sensory

53 tuning to those stimuli ${ }^{11,21}$. However, it is not known whether DA acting in the

54 sensory cortex itself could drive changes in the salience and incentive value of

55 sensory stimuli.

57 Here, we investigated the degree to which preferences for particular vocal

58 communication signals can be altered by manipulating neuromodulatory input to

59 the auditory cortex. We studied this in the zebra finch, a species of songbird in

60 which adult females identify individuals and select mates based on their complex,

61 learned vocalizations ('songs'). We found that pharmacological manipulation of 
62 dopaminergic activity in the auditory cortex significantly shaped preferences for

63 song and could reverse preferences for some songs over others. These data

64 suggest that DA can act directly in sensory processing areas to shape the

65 incentive salience of and preferences for stimuli.

67 RESULTS

68

69 Dopamine neurons in the caudal VTA show greater responses to preferred songs

70

71 We first quantified female preferences for songs using a two-choice operant

72 assay (Fig. 1A) ${ }^{22,23}$. In this assay, female zebra finches were provided two

73 strings, each of which activated the playback of a song from a single male zebra

74 finch when pulled (e.g., Male A for one string, Male B for the other string). For

75 each of the five song pairs tested, females showed significant song preferences

76 for one of the songs of the pair ( $p<0.01$ for all; Fig. 1B; see Methods). For four of

77 the five pairs, there was variation across females in which song was preferred

78 (e.g. some females preferred Male A, others Male B; Fig. 1C). However, for one

79 of the pairs of songs (Male $\mathrm{J}$ and Male $\mathrm{K}$ ), females consistently preferred the

80 song of one male of the pair over the song of the other male (Fig. $1 \mathrm{~B} ; \mathrm{t}_{(16)}=4.29$,

$81 \mathrm{p}=0.0006)$.

82 

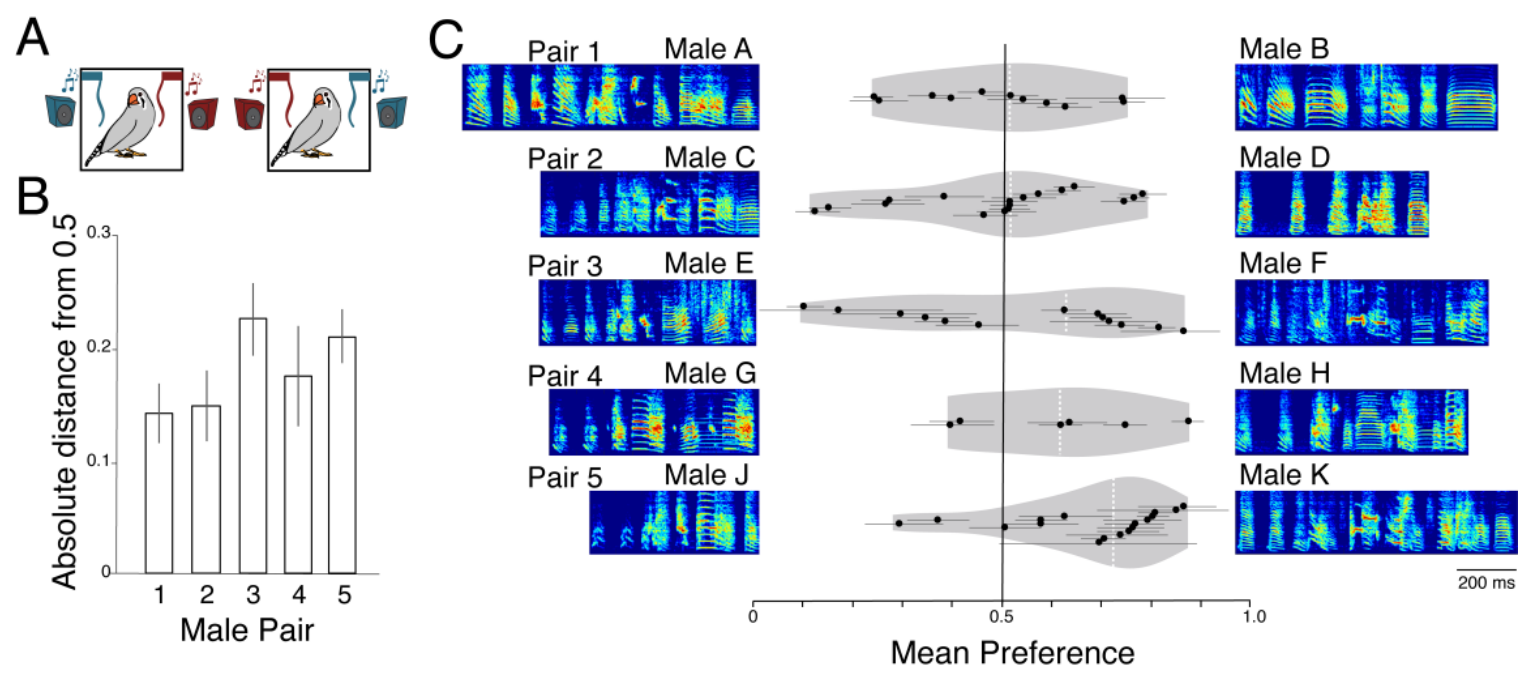

84 Figure 1. Females song preferences revealed by string pull assay. A) Females were tested in a string pull 85 assay where each string triggered playback of a song of a particular male. Contingencies were reversed halfway through testing to control for side bias. B) The absolute distance in preference score from 0.5 (which corresponds to a lack of bias for one specific song) was significantly greater than zero for all five pairs, indicating that females showed preferences for the song of one male over another. C) While each individual female showed significant preferences for one song within all pairs of male songs, there was significant individual variation in which male was preferred for all but one of the pairs. Violin plots (gray shading indicating the probability density) show the responses of females to pairs of songs, indicated by spectrograms of the song motifs at each end of the plots. Points are the responses of individual females with horizontal lines from the point indicating bootstrapped confidence intervals. Vertical white dashed lines indicate the median of preferences across all females. Motifs are labeled with an ID for each male and ordered by male pair as in B. Only for the bottom pair of males (Pair 5: Male $\mathrm{J}$ and Male K) did female preference differ significantly from 0.5 , indicating consistent preferences for Male $\mathrm{K}$ across females.

We leveraged the fact that a majority of females clearly preferred one of the two males in Pair 5 (Fig. 1C) to investigate whether catecholaminergic neurons in the midbrain and hindbrain differentially respond to songs with different degrees of

101 incentive salience, i.e. preferred versus less preferred songs. We calculated the 
108 cVTA expressed FOS following playback of preferred song than following

109 playback of the less-preferred song $(p=0.0013)$ or silence $(p=0.0002)$. In contrast,

110 while more $\mathrm{TH}$ neurons in the locus coeruleus (LC) and periaqueductal gray

111 (PAG) expressed FOS in response to hearing songs ( $p<0.0001$ for each), these

112 neurons responded similarly to preferred and less-preferred songs (PAG:

$113 \mathrm{p}=0.4943 ; \mathrm{LC}: \mathrm{p}=0.0630$ ). These data support previous work that finds that

114 catecholamine-synthesizing neurons in the midbrain and hindbrain respond to

115 playbacks of social signals ${ }^{24}$. In addition, these data highlight that dopaminergic

116 neurons in the VTA, but not in other catecholamine-producing cells in the

117 midbrain and hindbrain, are differentially activated by songs with different

118 degrees of incentive salience. Together, this suggests that songs with different

119 incentive values lead to differing amounts of dopamine release in areas

120 downstream to the VTA.

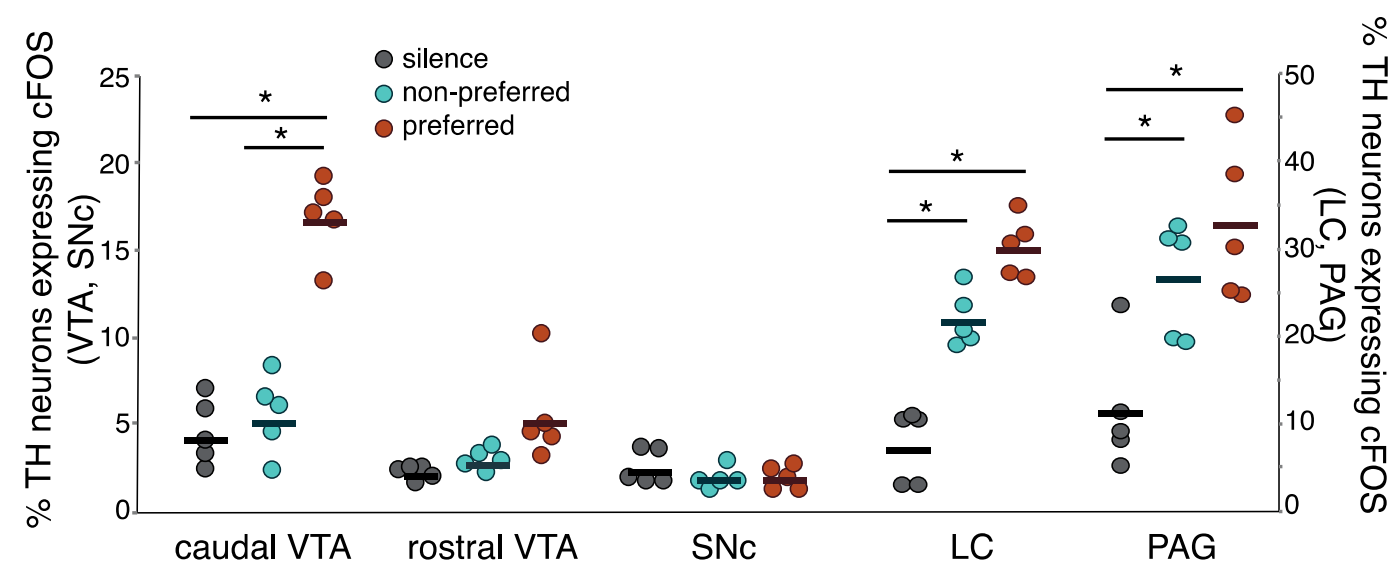

122 Figure 2. Hearing preferred songs drives FOS expression in dopaminergic neurons of the caudal

123 VTA. The percent of tyrosine hydroxylase $(\mathrm{TH})$ neurons expressing FOS in the ventral tegmental

124 area (VTA) and substantia nigra pars compacta (SNc; left axis) and the locus coeruleus (LC) and

125

126

127

128

129

130 periaqueductal gray (PAG; right axis) following playback of preferred songs (orange) or nonpreferred songs (teal) and in silent controls. In the caudal VTA, preferred song elicited significantly more FOS expression in TH neurons than either non-preferred song or silence. In the LC and PAG, both preferred and non-preferred songs elicited more FOS in TH neurons, however, there was no significant difference in the percent of TH neurons expressing FOS between preferred and non-preferred songs. 
132 Pairing song playback with a general dopamine agonist shifts song preference

134 To investigate whether catecholamine release into the auditory cortex could

135 shape song preferences, we paired infusions of catecholaminergic drugs into the

136 secondary auditory pallium (Fig. 3A and 3B) with passive playback of a male's

137 song. We targeted the caudomedial nidopallium (NCM), a secondary auditory

138 region important for auditory processing and implicated in auditory memory ${ }^{25-28}$.

139 In particular, after measuring the song preferences of individual females (Day 1),

140 we paired infusions of catecholamine agonists with playback of the less-preferred

141 song and paired infusions of vehicle (5\% DMSO in phosphate buffered saline

142 (PBS); see Methods) with playback of the preferred song (Days 2 and 3).

143 Females were then re-tested for song preferences (Day 4; i.e., preference for one

144 song compared to the other song of the pair; same as Day 1; see Methods; Fig.

145 3B).

147 We first tested the effects of broad, general catecholaminergic agonists to

148 determine whether DA or norepinephrine (NE) could alter female song

149 preferences (change in preference scores from Day 1 to Day 4). We found that

150 pairing the less-preferred song with the general DA agonist apomorphine (APO)

151 significantly affected female preferences for song $\left(t_{(5)}=-5.09, p=0.0038\right)$.

152 Specifically, pairing playbacks of the less-preferred song with APO infusions into

153 the NCM led to a significant increase in preference for that song between Day 1 
$157 t(6)=1.06, p=0.3306$, Fig 3D). Similarly, pairing the less-preferred song with NE in

158 the NCM also did not significantly alter preferences $\left(t_{(5)}=-1.52, p=0.1895\right.$; Fig.

159 3E).

A

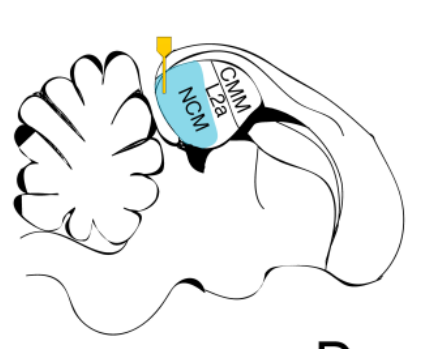

C

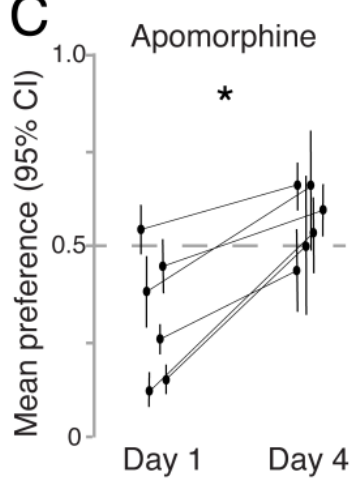

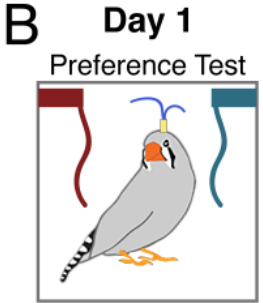

$D_{1.0} \quad$ Vehicle

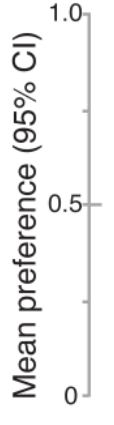

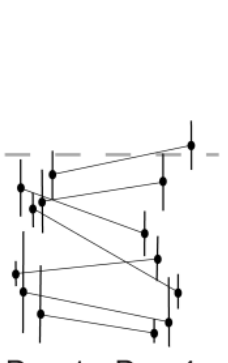

Day 1 Day 4

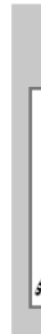

Day 2

Day 3 Song Playback + NCM Infusions
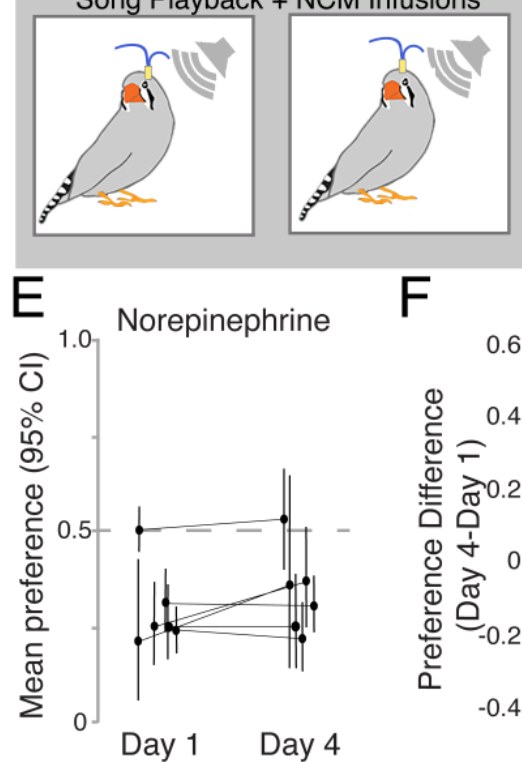

Day 4 Preference Test
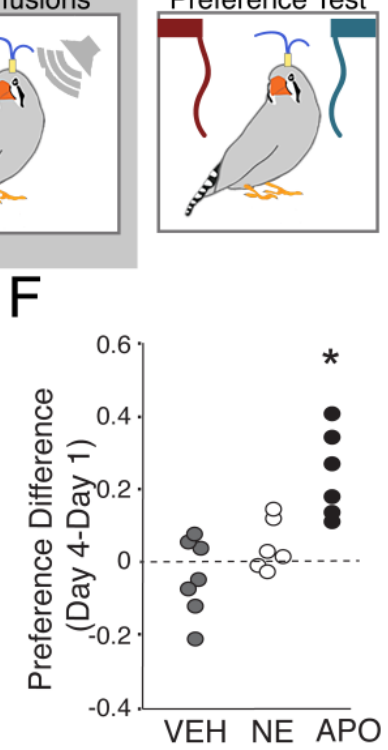

Figure 3. Dopamine in NCM modulates song preference. A) Microdialysis cannulae were implanted into the caudomedial nidopallium (NCM; blue). Parasagittal illustration of the location of the cannulae (yellow) relative to the NCM, the input "layer" of the primary auditory pallium Field L (L2a), and the secondary auditory pallial region the caudomedial mesopallium (CMM). Dorsal is up, caudal is left. B) Diagram of the experimental protocol. Females were tested for preference using a string pull assay on Day 1. On Days 2 and 3 females were infused with either vehicle (VEH) or drug 10 minutes prior to 2-hours of song playback. Drug and song combinations during Days 2 and 3 depended on the experiment (see Methods). In general, for agonist tests, agonists were paired with playback of the less-preferred song and VEH was paired with the preferred song. For control tests, VEH infusions were paired with playback of the less-preferred as well as the preferred songs. On Day 4, females were tested again in the string pull assay. The order of drug and control infusions (Day 2 and Day 3) were randomized across birds and tests. C) Females given the general agonist apomorphine paired with playback of the less-preferred song significantly shifted their preferences toward the originally less-preferred song. Points are the preference for the less-preferred song for individual birds, bars indicate bootstrapped confidence intervals (see Methods). No significant changes in preference from Day 1 to Day 4 were observed in vehicle treated control females(D) or in females given norepinephrine (E). F) The change in preference between Day 1 and Day 4 was significantly higher in females that received apomorphine (APO) than females infused with VEH or norepinephrine (NE). ${ }^{*}$ indicates $p<0.05$. 
180 To compare directly between drugs, we calculated the change in preference from

181 Day 1 to Day 4 and compared the degree of change between the three

182 treatments (Fig. 3F; see Methods). Overall, there was significant variation across

183 treatments $\left(\mathrm{F}_{(2,12.4)}=10.43, \mathrm{p}=0.0022\right)$, with a greater change in preference for

184 APO than for either NE $(p=0.0334)$ or $\operatorname{VEH}(p=0.0017)$. Thus, when coupled with

185 passive song playback, DA, but not NE, in a secondary auditory cortical region

186 modulates song preference. These data indicate that DA in the secondary

187 auditory cortex influences the incentive salience of songs. Given this finding, it is

188 important to reveal the involvement of specific DA receptor subtypes, persistence

189 of the effect, and nature of drug pairing on female auditory preferences.

D1 receptors participate in shifting song preference

193 D1-type receptors are highly expressed in the auditory forebrain, including

$194 \mathrm{NCM}^{29}$. To investigate the degree to which D1-type receptors are involved in

195 song preferences, we paired song playback with D1 receptor-specific agonists,

196 antagonists, or VEH (see Methods). Like APO, pairing infusions of the D1

197 receptor-specific agonist SKF81297 into the NCM with playback of the less-

198 preferred song led to a significant increase in the preference for the less-

199 preferred song $\left(\mathrm{t}_{(5)}=-5.10 ; \mathrm{p}=0.0038\right.$; Fig. 4A) such that females no longer

200 demonstrated a significant preference for the previously preferred song.

201 Conversely, pairing playback of the preferred song with infusions of the D1-

202 receptor antagonist $(\mathrm{SCH} 23390)$ tended to decrease the preference for the 
203 preferred song $(\mathrm{t}(6)=-1.84 ; \mathrm{p}=0.1147$; Fig. 4B). Both D1-receptor drugs produced

204 greater shifts in preference relative to the vehicle control condition $\left(F_{(2,6.62)}=45.31\right.$;

$205 p=0.0001$; Fig. 4C). In particular, the shift in preference was significantly greater

206 for the D1-receptor agonist than for $\operatorname{VEH}(p=0.0001)$, and tended to be greater for

207 the D1-antagonist than for $\mathrm{VEH}(\mathrm{p}=0.0541)$. Together these data indicate that

208 manipulation of D1 receptors in the auditory cortex during song playback can

209 produce substantial changes in preference and highlight the importance of D1

210 receptors in the auditory forebrain in ascribing incentive salience to stimuli.

A

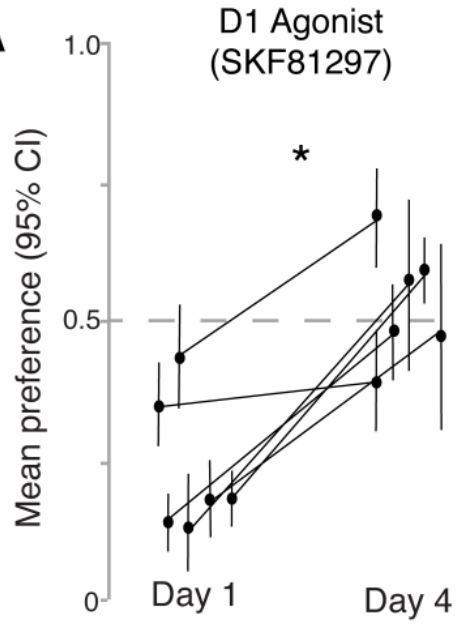

B

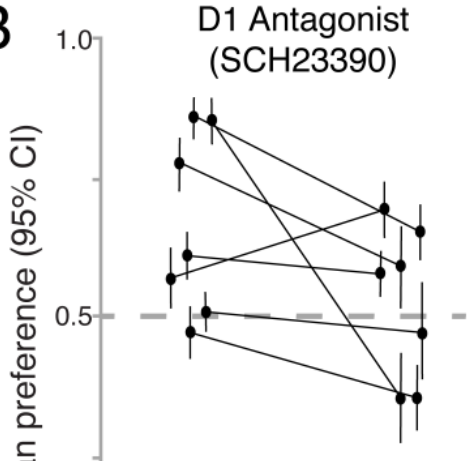

1 Antagonist

212

213

214

215

216

217

218

219

220

221

222

223 Given the known roles of dopamine in modulating motor behavior and motivation,

Figure 4. Dopamine D1 receptors in the NCM affect song preference A) Females given the D1 receptor agonist SKF81297 paired with playback of the less-preferred song significantly increased their preferences toward the originally less-preferred song. Points are individual birds, bars indicate bootstrapped confidence intervals (see Methods). No preference is at 0.5 indicated by the dashed line. B) Females given the D1 receptor antagonist SCH23390 paired with playback of the preferred song show a trend toward diminished preference for the preferred song. C) The change in preference between Day 1 and Day 4 was greater in females that received the D1 receptor agonist or antagonist than females infused with VEH. * indicates $p<0.05$; \# indicates $p=0.054$.

\section{Song preference shifts are not a consequence of changes in motor behavior}

224 we investigated whether the drug manipulations had general effects on string 
225 pulling behavior overall and the degree to which these changes could have

226 contributed to the shifts in preference. While across all treatments, the total

227 amount of string pulling for either string on Day 4 was lower than that on Day 1

$228\left(F_{(1,43.2)=8.78, p=0.0049)}\right.$, the shifts in preference were not due to the overall

229 changes in string pulling. Although different drugs had different effects on female

230 preferences, the degree to which females changed the amount of string pulling

231 did not vary across drugs (Drug X Day interaction: $F_{(4,43.2)}=0.49, p=0.7455$; Suppl

232 Fig. 1A). Further, the percent change in string pulling did not significantly

233 correlate with changes in preference for any of the conditions (Suppl Fig. 1B;

$234 p>0.05$ for all conditions). Taken together, these data indicate that the changes in

235 preference were not due to general effects of drug manipulations on motor

236 behavior or the motivation to hear song.

A

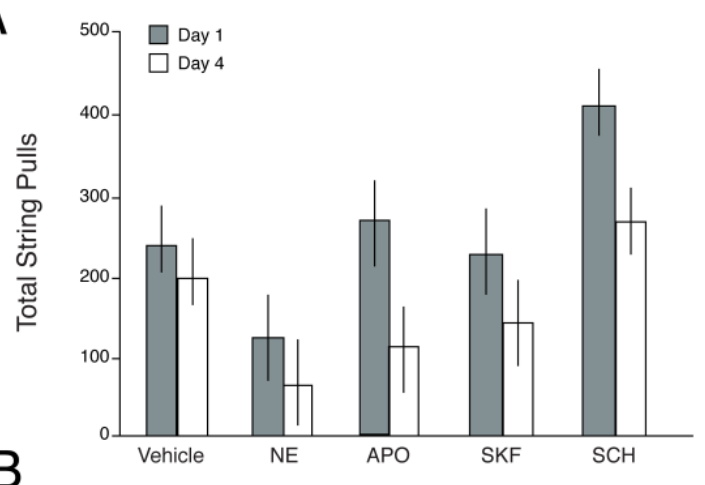

B
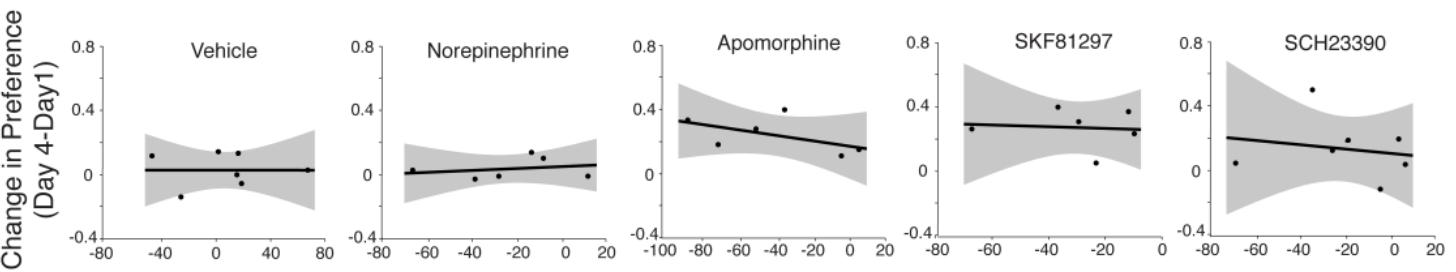

$\%$ Change in String Pulls

Suppl. Figure 1. Preference changes not correlated with general changes in motor behavior or motivation. A) Across all conditions, the total number of string pulls decreased between Day 1 and Day 4. Vehicle (VEH), norepinephrine (NE), apomorphine (APO), D1 agonist SKF81297 (SKF), D1 antagonist SCH23390 (SCH).

B) However, the percent change in string pulls was not significantly correlated with the change in preference 
$244 D A$ infusions into NCM lead to lasting changes in song preferences

245

246 To determine the degree to which manipulation of dopamine in the NCM can lead

247 to lasting changes in preference, we also tested a subset of birds one week

248 following pairing of song playback and the D1 agonist (see Methods). We found

249 that these females continued to showed preferences that were significantly

250 shifted from baseline (Fig. 5A). In particular, females tested one week after

251 pairing of the D1 receptor agonist and the less-preferred song had significantly

252 increased preferences for the previously less-preferred song $\left(t_{(5)}=-4.76\right.$,

$253 \mathrm{p}=0.0050)$. Moreover, the magnitude of the shift in preference was not

254 significantly different from females tested immediately following pairing of the D1

255 receptor agonist and the less-preferred song $\left(F_{(1,10)}=0.05 ; p=0.8371 ; F i g .5 B\right)$.

256 Thus, pairing of song playback with D1 receptor stimulation can result in lasting

257 changes in song preference.

A

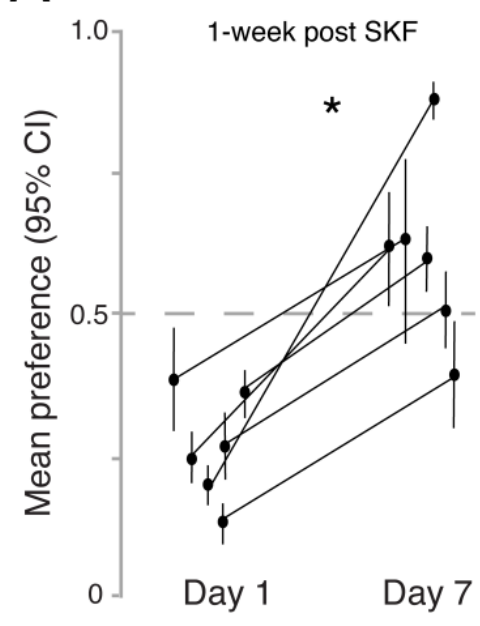

B

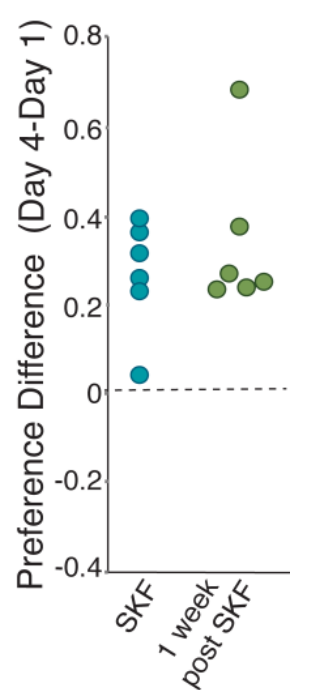


showed significant increases in preference for the less-preferred song. Points are individual birds, bars indicate bootstrapped confidence intervals (see Methods). No preference is at 0.5 indicated by the dashed line. B) Preference changes induced by D1 agonist paired with playback of the less-preferred song for females tested on Day 4 ("SKF"; teal) did not differ significantly from the preference changes of females tested 1 week later (green). " "* indicates $p<0.05$.

268 playback

270 The effects of VTA stimulation on tonotopic maps in the primary auditory cortex

271 depend on the timing of stimulation relative to sound playback ${ }^{19}$. This

272 suggests the possibility that D1-receptor-mediated changes in preference may

273 require a temporal correspondence between drug infusion and song playback. To

274 investigate the importance of the temporal association between drug infusion and

275 song playback for changes in preference, we uncoupled the timing of drug

276 delivery and song exposure and estimated changes to song preferences (see

277 Methods). Specifically, during the song exposure on either Day 2 and 3, females

278 were infused with the D1 agonist for 2-hrs beginning 15-30 minutes after the

279 termination of playback of the less-preferred song. This uncoupling of drug

280 infusion and song playback did not lead to a significant shift in song preferences

$281\left(t_{(5)}=1.11, p=0.3181\right.$; Fig. $\left.6 A\right)$. Moreover, the change in preference when the D1-

282 receptor agonist was uncoupled from song was significantly less than when the

283 D1-receptor agonist was coupled with song playback ( $p=0.0033$; Fig. 6B), and

284 not significantly different than the lack of preference change following VEH

$285(p=0.8283)$. Together, these data indicate that the reorganization of song

286 preference requires the temporal coupling of D1-receptor activation and song

287 playback. 


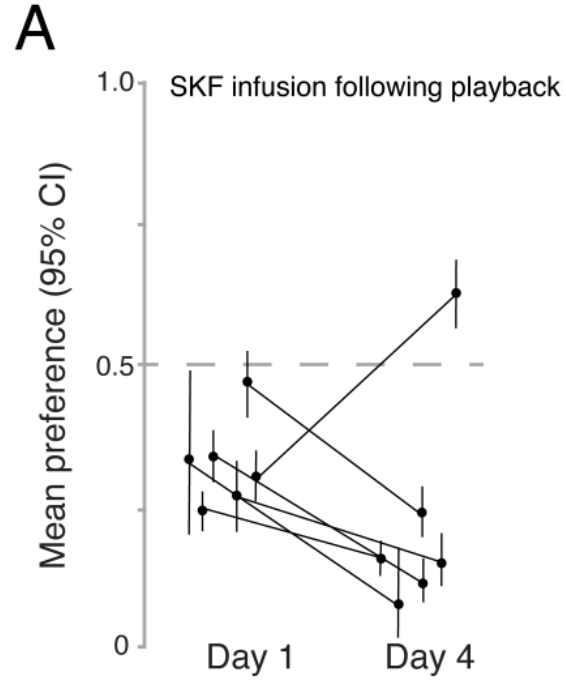

Day 1

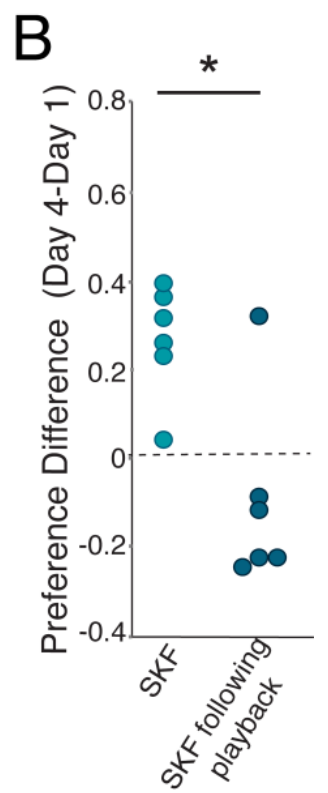

Figure 6. Preference shifts are dependent upon the timing of drug infusion. A) Infusing the D1 receptor agonist into NCM after song playback did not lead to greater preference for the less-preferred song. Points are individual birds, bars indicate bootstrapped confidence intervals (see Methods). B) Comparison of preference changes induced by D1 agonist paired with playback of the less-preferred song for females tested on Day 4 ("SKF"; teal) and of females that received SFK infusions 15-30 minutes following song playback (blue). "** indicates $p<0.05$.

295

DISCUSSION

298 In vocal communication systems, receiver preferences for vocal signals can

299 depend not only on the features of the signal but also on the receiver's individual

300 experience or internal state. The dual tasks of processing a signal's acoustic

301 features and its incentive value have been postulated to rely on physiological

302 processes within sensory and reinforcement pathways respectively. In particular,

303 auditory cortical circuits are thought to be responsible for detecting and

304 discriminating acoustic signals while the nucleus accumbens and VTA ascribe

305 salience and modulate preference for those signals. In line with this, we found

306 that in female zebra finches preferred songs elicit greater activity in dopamine 
307 neurons of the caudal VTA than less-preferred songs. However, we also found

308 that coupling song playback with pharmacological manipulations of dopamine

309 receptors within the auditory cortex itself could alter and, in some cases, fully

310 reverse song preferences. The changes to preference were lasting, as females

311 still displayed the reversed preference one week later. Moreover, the changes to

312 preference were not just a consequence of dopamine-dependent changes in the

313 auditory cortex, since females that heard playback before drug manipulation did

314 not show altered preferences. Taken together, these data indicate that

315 dopaminergic projections to the auditory forebrain may directly modulate

316 behaviorally-relevant auditory preferences and motivate behavior in response to

317 vocal signals.

319 Pairing DA agonists in the NCM with playback of a less-preferred song resulted

320 in increased preferences for the less-preferred stimulus. Thus, song preferences

321 are not only plastic under the right conditions, but they can be altered by changes

322 in the auditory forebrain. In rats, stimulation of the VTA leads to changes in the

323 inhibitory tone and plasticity in the tonotopic organization of primary auditory

324 cortex (A1) ${ }^{19,30}$. Specifically, VTA stimulation enhances circuit inhibition in A1,

325 thereby increasing the auditory-evoked firing precision of $A 1$ neurons ${ }^{30}$.

326 Moreover, pairing VTA stimulation with playback of a tone leads to expanded

327 representation of that tone in $A 1^{19}$. Such targeted changes to $A 1$ firing precision

328 and tonotopic organization are associated with increased ability to discriminate

329 sounds ${ }^{31-33}$. However, while previous studies have shown that VTA stimulation 
330 leads to auditory cortical plasticity and improved discrimination, changes just to

331 the representation of a sound have not been hypothesized to result in changes in

332 preferences. Indeed, in our study, birds were not simply discriminating between

333 two stimuli, but were pulling strings to hear one song over another; therefore,

334 their string pulling behavior provided a read-out of their motivation to hear a

335 particular stimulus. Thus, our data indicate that increasing dopaminergic activity

336 in the auditory forebrain could lead not only to an increase in the signal-to-noise

337 ratio, as seen in rodents, but also to a change in the motivation to hear the song

338 or the pleasure derived from it.

340 At the same time, we found that preference for a preferred song could be

341 diminished following pairing of playback with infusion of a D1-receptor antagonist.

342 Thus, our data support the possibility that dopamine is released into the NCM in

343 response to preferred songs and this release may be important for the sustained

344 preference for that song. These data dovetail with previous work demonstrating

345 that, in female sparrows, hearing conspecific song (versus silence) leads to

346 increases in the expression of phosphorylated tyrosine hydroxylase, a marker of

347 dopamine synthesis, in the $\mathrm{NCM}$ and $\mathrm{CMM}^{34}$. Together with the lower expression

348 of FOS in response to less-preferred songs, this indicates that least preferred

349 songs may elicit lower levels of dopamine release. Future studies using online

350 methods to measure local changes in dopamine release ${ }^{35-37}$ in the auditory

351 cortex in response to songs of different perceived quality will provide needed

352 data to clarify this relationship. 
354 Dopamine release in the striatum and the cortex leads to plasticity through both

355 long-term potentiation (LTP) and depression (LTD) ${ }^{38-42}$. For example, in the

356 songbird basal ganglia nucleus Area X, induction of LTP requires activation of

357 both NMDA and D1 receptors ${ }^{38}$, while in the rat prefrontal cortex dopamine

358 lowers the threshold for LTD $^{42}$. The effects of dopamine receptor activation on

359 synaptic plasticity in primary sensory areas have not been directly addressed,

360 however, one interesting possibility is that, through similar synaptic mechanisms,

361 pairing of song and dopamine stimulation may lead to changes in the encoding of

362 auditory objects that could lead to enhanced memory of the song ${ }^{43,44}$. The NCM

363 has been implicated in auditory memory for songs, including memories of the

364 song of the tutor as well as a mate $26,45,46$. Moreover, female songbirds show long-

365 lasting memory for the songs of specific familiar individuals, including songs of

366 the tutor and the mate $26,47-49$. In males, NCM neurons become more selective for

367 the tutor song following tutoring ${ }^{27}$. Whether similar processes occur in females,

368 and the degree to which they are dopamine-dependent is unknown. Future

369 investigation of whether dopamine in the NCM not only modulates song

370 preference but also leads to the formation of auditory memories for especially

371 salient or preferred songs, such as the mate's song, will provide needed and

372 novel insight into the function of the NCM in auditory perception.

373

374 Attribution of social salience to acoustic signals is a critical step in auditory

375 processing for vocal communicators. Our data extend existing knowledge about 
376 catecholamine function in the auditory cortex ${ }^{15,19,30,50,51}$. Moreover, our results

377 implicate the auditory cortex in the shaping of auditory preferences. Thus,

378 dopamine-dependent changes in the auditory cortex may not only increase

379 representational distinctiveness, heighten signal-to-noise, and improve

380 discrimination ability, as has been seen in studies on rodents, but these changes

381 can lead directly to a change in the incentive salience of the sound.

382

383 METHODS

385 Animals

386 All zebra finch females used in this study ( $\mathrm{N}=36,>90$ days post-hatch) were

387 raised with both parents and all siblings until 60 days of age. Thereafter, they

388 were housed in same-sex group cages in a colony, and thus were acoustically

389 exposed to the vocalizations of both males and females. Females were

390 maintained on a 14-hour light, 10-hour dark schedule with ad libitum access to

391 seed, water, and grit. Lettuce and egg supplements were provided once per

392 week. Bird care and procedures followed all Canadian Council on Animal Care

393 guidelines and were approved by the Animal Care Committee of McGill

394 University.

395

396 Drugs

397 All drugs were dissolved in DMSO and diluted in sterile phosphate buffered

398 saline (PBS) such that the final concentration of DMSO was $5 \%$. We used three 
399 drugs that target dopamine receptors: Apomorphine (APO, a general dopamine

400 agonist; 3.3mM; Tocris Bioscience, Minneapolis, MN), SKF-81297 (1mM; a

401 selective D1-receptor agonist; Sigma Aldrich, Oakville, ON), SCH-23390 (1 mM, a

402 selective D1-receptor antagonist, Tocris Bioscience, Minneapolis, MN). We also

403 assessed the effects of norepinephrine (NE; $1 \mathrm{mM}$; Sigma Aldrich, Oakville, ON)

404 on song preferences. Drug concentrations were determined based on the

405 literature and personal communication ${ }^{52-56}$. For all tests, PBS containing $5 \%$

406 DMSO was used as the control vehicle solution (VEH).

407

408 Surgery

409 To manipulate catecholamine levels in NCM, females were bilaterally implanted

410 with microdialysis guide cannulae targeted at the NCM (Fig 3A). At least 30

411 minutes prior to surgical procedures, females were given an analgesic (Metacam,

412 company) and deprived of food and water. At the start of surgery, females

413 received an intramuscular injection of ketamine $(0.04 \mathrm{mg} / \mathrm{g})$ and midazolam

$414(0.0015 \mathrm{mg} / \mathrm{g})$ for anesthetic induction and then fitted into a stereotaxic apparatus

415 (Leica) with a fixed beak angle of 45 degrees. Once birds were placed into the

416 stereotaxic apparatus, anesthesia was maintained on $0-2 \%$ isoflurane vapor for

417 the duration of the surgery. Guide cannulae containing dummy probes (CMA/7,

418 CMA Microdialysis, Stockholm, Sweden) were implanted bilaterally in the NCM

419 (from the caudal Y-sinus: $50 \mu \mathrm{m}$ rostral, $50 \mu \mathrm{m}$ lateral, $150 \mu \mathrm{m}$ deep) through

420 small windows in both layers of skull and secured in place using epoxy and 
421 dental cement. Following surgery, all females were housed individually and given

422 at least a week to recover before beginning retrodialysis and behavior testing.

423

424 Reverse microdialysis

425 Females were fitted with microdialysis probes into the guide cannulae and

426 infused with VEH at least 24 hours before the start of the experiment to allow for

427 habituation. Solutions were retrodialyzed into the NCM using untethered

428 microdialysis probes (CMA Microdialysis, Kista, Sweden; pore size 6,000

429 Daltons). Specifically, probe input and output tubing were trimmed to 3-4 cm and

430 fitted with connectors and custom-made stoppers. Outside of the experimental

431 period, females were infused every 12 hours with VEH using a syringe pump

432 (Harvard Apparatus, Holliston, MA; $10 \mu \mathrm{l} / \mathrm{min}$ for 4 minutes). On infusion days

433 (Days 2 and 3), tubing was filled using the syringe pump with 40 ul of drug or

434 VEH via the input tubing. Following song playback exposure, tubing was flushed

435 with VEH $(10 \mu \mathrm{l} / \mathrm{min}$ for 4 minutes) then filled with $40 \mathrm{ul}$ of $\mathrm{VEH}(10 \mu \mathrm{l} / \mathrm{min}$ for 4

436 minutes).

$438 \quad$ Preference testing

439 For the duration of testing, females were individually housed in sound-attenuating

440 chambers (TRA Acoustics, Cornwall, Ontario) inside cages equipped to test song

441 preferences with a string-pull assay. Specifically, cages contained two Cherry $1 \mathrm{~g}$

442 levers, each with a piece of a burlap string attached. Levers were connected to a

443 computer via a connector block (National Instruments). Sound Analysis Pro 
444 software was used to record string pulls and playback songs ${ }^{22,57}$. During song

445 preference testing, levers were activated so that each string, when pulled,

446 triggered the playback of one male's song. For example, String 1 triggered the

447 playback of Male A's song, and String 2 triggered the playback of Male B's song

448 (Fig 3B). Preference tests consisted of two 2-hour sessions. Females were

449 required to pull each string a minimum of three times to initiate each session. For

450 the second session, the song triggered by each string was switched (i.e.,

451 contingencies reversed; for example, now String 1 triggered Male B's song, and

452 String 2 triggered Male A's song) to control for place/string preference. Following

453 the switch in contingencies, the second session began once the female had

454 pulled each string a minimum of three times. At the end of the second session, all

455 strings were removed from the cage.

456

457 Experimental Design

458 The experiment followed a four-day schedule. On Day 1, a female's preference

459 between two male songs was tested using the string pull assay, allowing us to

460 identify the "preferred song" and "less-preferred song." On Days 2 and 3, females

461 received retrodialysis of drug or $\mathrm{VEH}$ (one treatment per day) during two hours of

462 passive exposure to the songs of one of the males. The order of song exposure

463 (preferred vs. less-preferred songs) on days 2 and 3 was randomized within each

464 female across multiple experiments as well as between females. For tests using

465 DA and NE agonists, we paired playback of the less-preferred song with infusion

466 of either a DA receptor agonist (APO, SKF-81297) or NE, and paired playback of 
467 the preferred song with infusion of VEH. All infusions occurred 10 to 30 minutes

468 prior to the beginning of playbacks, and all drugs were washed out $(10 \mu \mathrm{l} / \mathrm{min}$ for

4694 minutes) within 30 minutes following the end of playbacks. On Day 4 , the

470 female's preference was retested (Fig 3C). For tests assessing whether DA

471 antagonism could decrease preference for the preferred song, we paired

472 playback of the preferred song with infusion of $\mathrm{SCH}-23390$ and playback of the

473 less-preferred song with VEH. In the control test, VEH was infused prior to

474 playback of both the preferred and less-preferred songs. Females each

475 underwent multiple experiments, with different pairs of male songs for each drug

476 manipulation. The pair of males paired with each drug manipulation and the order

477 of drug manipulation was randomized across females.

479 We also tested whether DA manipulation was necessary during the song

480 playback in order to affect preferences. In a separate set of experiments, the DA

481 agonist SKF81297 was infused 15-30 minutes after playback of the less-

482 preferred song.

484 Anatomy

485 Following the completion of experiments, birds were deeply anesthetized with

486 isoflurane before being transcardially perfused with $0.9 \%$ saline, followed by $4 \%$

487 paraformaldehyde in $0.025 \mathrm{M}$ phosphate buffer (PB). Brains were post-fixed for

488 4-hrs, then cryoprotected in 30\% sucrose. Forty-micron parasagittal sections

489 were cut on a freezing microtome and every third section was stained with cresyl 
490 violet acetate to determine the locations of cannulae and probes. All females in

491 this study were confirmed to have probes located within NCM.

493 Song stimuli

494 All male song stimuli were female-directed courtship song samples recorded from

495 males $(\mathrm{N}=10)$ from our colony at McGill University. Songs were recorded in

496 sound-attenuating chambers (TRA Acoustics, Cornwall, Ontario) by briefly

497 exposing males to stimulus females (not used in this experiment), as has been

498 previously described $22,26,58$. We created stimuli for five pairs of males for use in

499 two-choice female preference tests. Pairs consisted of the same two males for all

500 females (e.g. Male A and Male B were always pair 1, Male C and Male D were

501 always pair 2, etc.), and females were tested on 2-5 pairs. Males used to

502 generate song stimuli and females used in this study were unrelated and had

503 never physically interacted. Song stimuli used for the preference test were

504 matched for duration and number of introductory notes and were free of noise

505 and female calls. For each male, we used one song example containing multiple

506 motifs and introductory notes. All stimulus songs were bandpass filtered (300-10

$507 \mathrm{kHz})$, normalized by their maximum amplitude, and saved as wav files $(44.1 \mathrm{kHz})$

508 using custom written code in Matlab (Mathworks, Natick, MA). We used a

509 selection of 4-7 recordings of song to provide a representative sample of varying

510 song duration, number of bouts, and number of introductory notes from each

511 male's repertoire.

512 


\section{Analyses}

514 All statistical analyses were completed using JMP Statistical Processing Software

515 (SAS, Cary, NC, USA) or custom-written Matlab code (Mathworks, Natick, MA).

516 To quantify female preference for one song over the other, we determined the

517 distribution of string pulls for Male A versus Male B. The initially preferred male

518 was attributed a value of 1 , and the less-preferred male a value of zero, and the

519 distribution of pulls was bootstrapped with replacement (10,000 iterations) to

520 obtain $95 \%$ confidence intervals. We also calculated the "strength of preference"

521 (the distance of the bootstrapped distribution mean from 0.5, a chance

522 distribution). This allowed us to separate the strength of preference from the

523 directionality, for example if females had strong preferences overall but differed in

524 which male they preferred.

525

526 To assess whether females demonstrated song preferences during the

527 preference tests, we conducted two-tailed single-sample t-tests on mean

528 "strength of preference" for each pair of males and tested whether the distribution

529 of preference significantly differed from chance $(\mathrm{HO}=0.5)$. We also tested whether

530 mean preference between the two males was skewed in any of our pairs by

531 attributing one male a value of one and the other male a value of zero across all

532 females. We then conducted two-tailed single-sample t-tests by pair to see

533 whether the mean preference between two males was significantly different from

5340.5 (chance).

535 
536 To examine whether the drug manipulation paired with playback of the less-

537 preferred male's song was related to change in preference between Day 1 and

538 Day 4, we performed paired t-tests of the bootstrapped means for Day 1 and Day

5394 for each drug. We also tested whether the amount by which behavior shifted

540 was related to drug manipulation using a model with percent change in string pull

541 distribution as a dependent variable, drug as an independent variable, and

542 female ID as a random variable. All models were conducted using a restricted

543 maximum likelihood approach with unbounded variance components.

544

545 Finally, we examined whether overall changes in activity could account for the

546 changes in preference. To do this, we used a model with percent change in string

547 pull distribution as the dependent variable, percent change in overall string

548 pulling as an independent variable, and bird ID as a random variable,

549 independently for each drug.

550

551 ACKNOWLEDGEMENTS

552 We would like to thank Jon T. Sakata for helpful discussions and comments on

553 the manuscript. In addition, we would like to thank Therese Koch for help with

554 preference testing and histology. This work was funded by the Natural Sciences

555 and Engineering Research Council of Canada (NSERC) and Fonds de

556 Recherche du Québec - Nature et technologies (FQRNT) to SCW, a McGill

557 Integrated Program in Neuroscience returning student award to HJB, and a Lloyd

558 Carr-Harris fellowship to EMW. 
560 AUTHOR CONTRIBUTIONS

561 HJB and SCW designed the experiments; HJB and EMW performed the

562 experiments; HJB and SCW analyzed the data; HJB, EMW, and SCW wrote and

563 edited the manuscript.

564

565 COMPETING INTERESTS

566 The authors declare no competing interests.

567

568 REFERENCES

569 1. Bakin, J. S., South, D. A. \& Weinberger, N. M. Induction of receptive field

570 plasticity in the auditory cortex of the guinea pig during instrumental

571 avoidance conditioning. Behavioral Neuroscience 110, 905-913 (1996).

572 2. Edeline, J.-M., Pham, P. \& Weinberger, N. M. Rapid development of learning-

573 induced receptive field plasticity in the auditory cortex. Behavioral

$574 \quad$ Neuroscience 107, 539-551 (1993).

575 3. Froemke, R. C. \& Jones, B. J. Development of auditory cortical synaptic

576 receptive fields. Neuroscience \& Biobehavioral Reviews 35, 2105-2113

$577 \quad(2011)$

578 4. Pienkowski, M. \& Eggermont, J. J. Cortical tonotopic map plasticity and

579 behavior. Neuroscience \& Biobehavioral Reviews 35, 2117-2128 (2011).

580 5. Miranda, J. A. \& Wilczynski, W. Female reproductive state influences the

581 auditory midbrain response. J Comp Physiol A 195, 341-349 (2009). 
582 6. Sisneros, J. A. Steroid-dependent auditory plasticity for the enhancement of

583 acoustic communication: Recent insights from a vocal teleost fish. Hearing

$584 \quad$ Research 252, 9-14 (2009).

585 7. Caras, M. L., Sen, K., Rubel, E. W. \& Brenowitz, E. A. Seasonal Plasticity of

586 Precise Spike Timing in the Avian Auditory System. J. Neurosci. 35, 3431-

$587 \quad 3445(2015)$.

588 8. Miranda, J. A. \& Liu, R. C. Dissecting natural sensory plasticity: Hormones

589 and experience in a maternal context. Hearing Research 252, 21-28 (2009).

590 9. Marlin, B. J., Mitre, M., D’amour, J. A., Chao, M. V. \& Froemke, R. C.

591 Oxytocin enables maternal behaviour by balancing cortical inhibition. Nature

$592 \quad 520,499-504(2015)$.

593 10. Marlin, B. J. \& Froemke, R. C. Oxytocin modulation of neural circuits for

594 social behavior. Developmental Neurobiology 77, 169-189 (2017).

595 11. Maney, D. L. The incentive salience of courtship vocalizations: Hormone-

596 mediated 'wanting' in the auditory system. Hearing Research 305, 19-30

597 (2013).

598 12. Berridge, K. C. The debate over dopamine's role in reward: the case for

599 incentive salience. Psychopharmacology 191, 391-431 (2007).

600 13. Berke, J. D. What does dopamine mean? Nature Neuroscience 21, 787-793

$601 \quad$ (2018).

602 14. Schultz, W., Dayan, P. \& Montague, P. R. A Neural Substrate of Prediction

603 and Reward. Science 275, 1593-1599 (1997). 
604 15. Bromberg-Martin, E. S., Matsumoto, M. \& Hikosaka, O. Dopamine in

605 Motivational Control: Rewarding, Aversive, and Alerting. Neuron 68, 815-834

$606 \quad$ (2010).

607 16. Fields, H. L., Hjelmstad, G. O., Margolis, E. B. \& Nicola, S. M. Ventral

608 Tegmental Area Neurons in Learned Appetitive Behavior and Positive

609 Reinforcement. Annual Review of Neuroscience 30, 289-316 (2007).

610 17. Aragona, B. J., Liu, Y., Curtis, J. T., Stephan, F. K. \& Wang, Z. A Critical Role

611 for Nucleus Accumbens Dopamine in Partner-Preference Formation in Male

612 Prairie Voles. J. Neurosci. 23, 3483-3490 (2003).

613 18. Gobrogge, K. \& Wang, Z. The ties that bond: neurochemistry of attachment in 614 voles. Current Opinion in Neurobiology 38, 80-88 (2016).

615 19. Bao, S., Chan, V. T. \& Merzenich, M. M. Cortical remodelling induced by

616 activity of ventral tegmental dopamine neurons. Nature 412, 79 (2001).

617 20. Puschmann, S., Brechmann, A. \& Thiel, C. M. Learning-dependent plasticity

618 in human auditory cortex during appetitive operant conditioning. Human Brain

619 Mapping 34, 2841-2851 (2013).

620 21. Salimpoor, V. N., Zald, D. H., Zatorre, R. J., Dagher, A. \& Mclntosh, A. R.

621 Predictions and the brain: how musical sounds become rewarding. Trends in

622 Cognitive Sciences 19, 86-91 (2015).

623 22. Schubloom, H. E. \& Woolley, S. C. Variation in social relationships relates to 624 song preferences and EGR1 expression in a female songbird. Developmental $625 \quad$ Neurobiology 76, 1029-1040 (2016). 
626 23. Riebel, K., Smallegange, I. M., Terpstra, N. J. \& Bolhuis, J. J. Sexual equality

627 in zebra finch song preference: evidence for a dissociation between song

628 recognition and production learning. Proceedings of the Royal Society of

629 London. Series B: Biological Sciences 269, 729-733 (2002).

630 24. Barr, H. J. \& Woolley, S. C. Developmental auditory exposure shapes

631 responses of catecholaminergic neurons to socially-modulated song.

632 Scientific Reports 8, (2018).

633 25. Bolhuis, J. J. \& Gahr, M. Neural mechanisms of birdsong memory. Nature

634 Reviews Neuroscience 7, 347-357 (2006).

635 26. Woolley, S. C. \& Doupe, A. J. Social Context-Induced Song Variation Affects

636 Female Behavior and Gene Expression. PLOS Biology 6, e62 (2008).

637 27. Yanagihara, S. \& Yazaki-Sugiyama, Y. Auditory experience-dependent

638 cortical circuit shaping for memory formation in bird song learning. Nature

639 Communications 7, 11946 (2016).

640 28. Chew, S. J., Vicario, D. S. \& Nottebohm, F. A large-capacity memory system

641 that recognizes the calls and songs of individual birds. Proceedings of the

$642 \quad$ National Academy of Sciences 93, 1950-1955 (1996).

643 29. Kubikova, L., Wada, K. \& Jarvis, E. D. Dopamine receptors in a songbird

644 brain. The Journal of Comparative Neurology 518, 741-769 (2010).

645 30. Lou, Y. et al. Ventral tegmental area activation promotes firing precision and 646 strength through circuit inhibition in the primary auditory cortex. Front. Neural

647 Circuits 8, (2014). 
648 31. Shepard, K. N., Kilgard, M. P. \& Liu, R. C. Experience-Dependent Plasticity

649 and Auditory Cortex. in Neural Correlates of Auditory Cognition (eds. Cohen,

650 Y. E., Popper, A. N. \& Fay, R. R.) 293-327 (Springer New York, 2013).

651 doi:10.1007/978-1-4614-2350-8_10

652 32. Reed, A. et al. Cortical Map Plasticity Improves Learning but Is Not

$653 \quad$ Necessary for Improved Performance. Neuron 70, 121-131 (2011).

654 33. Carcea, I. \& Froemke, R. C. Chapter 3 - Cortical Plasticity, Excitatory-

655 Inhibitory Balance, and Sensory Perception. in Progress in Brain Research

656 (eds. Merzenich, M. M., Nahum, M. \& Van Vleet, T. M.) 207, 65-90 (Elsevier, 657 2013).

658 34. Matragrano, L. L. et al. Rapid Effects of Hearing Song on Catecholaminergic 659 Activity in the Songbird Auditory Pathway. PLoS ONE 7, e39388 (2012).

660 35. Tanaka, M., Sun, F., Li, Y. \& Mooney, R. A mesocortical dopamine circuit 661 enables the cultural transmission of vocal behaviour. Nature 563, 117-120 662 (2018).

663 36. Hamid, A. A. et al. Mesolimbic dopamine signals the value of work. Nature $664 \quad$ Neuroscience 19, 117-126 (2016).

665 37. Howe, M. W. \& Dombeck, D. A. Rapid signalling in distinct dopaminergic 666 axons during locomotion and reward. Nature 535, 505-510 (2016).

667 38. Ding, L. \& Perkel, D. J. Long-Term Potentiation in an Avian Basal Ganglia 668 Nucleus Essential for Vocal Learning. J. Neurosci. 24, 488-494 (2004).

669 39. Ding, L. \& Perkel, D. J. Dopamine Modulates Excitability of Spiny Neurons in 670 the Avian Basal Ganglia. J. Neurosci. 22, 5210-5218 (2002). 
671 40. Calabresi, P., Picconi, B., Tozzi, A. \& Di Filippo, M. Dopamine-mediated

672 regulation of corticostriatal synaptic plasticity. Trends in Neurosciences $\mathbf{3 0}$,

$673 \quad 211-219(2007)$.

674 41. Otani, S., Daniel, H., Roisin, M.-P. \& Crepel, F. Dopaminergic Modulation of

675 Long-term Synaptic Plasticity in Rat Prefrontal Neurons. Cereb Cortex 13,

$676 \quad 1251-1256(2003)$.

677 42. Sheynikhovich, D., Otani, S. \& Arleo, A. Dopaminergic Control of Long-Term

678 Depression/Long-Term Potentiation Threshold in Prefrontal Cortex. J.

$679 \quad$ Neurosci. 33, 13914-13926 (2013).

680 43. Wise, R. A. Dopamine, learning and motivation. Nature Reviews

$681 \quad$ Neuroscience 5, 483-494 (2004).

682 44. Lisman, J., Grace, A. A. \& Duzel, E. A neoHebbian framework for episodic

683 memory; role of dopamine-dependent late LTP. Trends in Neurosciences 34,

$684 \quad 536-547(2011)$.

685 45. London, S. E. \& Clayton, D. F. Functional identification of sensory

686 mechanisms required for developmental song learning. Nature Neuroscience

$687 \quad 11,579-586(2008)$.

688 46. Gobes, S. M. H. \& Bolhuis, J. J. Birdsong Memory: A Neural Dissociation

689 between Song Recognition and Production. Current Biology 17, 789-793

$690 \quad$ (2007).

691 47. Miller, D. B. The acoustic basis of mate recognition by female zebra finches.

692 anim behav 27, 376-380 (1979). 
693 48. Miller, D. B. Long-term recognition of father's song by female zebra finches.

$694 \quad$ Nature 280, 389-391 (1979).

695 49. Clayton, N. S. Song discrimination learning in zebra finches. Animal

696 Behaviour 36, 1016-1024 (1988).

697 50. Happel, M. F. K. Dopaminergic impact on local and global cortical circuit

698 processing during learning. Behavioural Brain Research 299, 32-41 (2016).

699 51. Sara, S. J. \& Bouret, S. Orienting and Reorienting: The Locus Coeruleus

$700 \quad$ Mediates Cognition through Arousal. Neuron 76, 130-141 (2012).

701 52. Acerbo, M. J. \& Delius, J. D. Behavioral Sensitization to Apomorphine in

702 Pigeons (Columba livia): Blockade by the $\mathrm{D}_{1}$ Dopamine Antagonist SCH-

703 23390. Behavioral Neuroscience 118, 1080-1088 (2004).

704 53. Rose, J., Schiffer, A.-M., Dittrich, L. \& Güntürkün, O. The role of dopamine in

705 maintenance and distractability of attention in the "prefrontal cortex" of

$706 \quad$ pigeons. Neuroscience 167, 232-237 (2010).

707 54. Herold, C., Diekamp, B. \& Güntürkün, O. Stimulation of dopamine D1

708 receptors in the avian fronto-striatal system adjusts daily cognitive

709 fluctuations. Behavioural Brain Research 194, 223-229 (2008).

710 55. Diekamp, B., Kalt, T., Ruhm, A., Koch, M. \& Güntürkün, O. Impairment in a

711 discrimination reversal task after D1 receptor blockade in the pigeon

712 'prefrontal cortex'. Behavioral Neuroscience 114, 1145-1155 (2000).

713 56. Ikeda, M. Z., Jeon, S. D., Cowell, R. A. \& Remage-Healey, L. Norepinephrine

714 Modulates Coding of Complex Vocalizations in the Songbird Auditory Cortex 
715 Independent of Local Neuroestrogen Synthesis. Journal of Neuroscience 35,

$716 \quad 9356-9368(2015)$.

717 57. Tchernichovski, O., Nottebohm, F., Ho, C. E., Pesaran, B. \& Mitra, P. P. A

718 procedure for an automated measurement of song similarity. Animal

719 Behaviour 59, 1167-1176 (2000).

720 58. Chen, Y., Clark, O. \& Woolley, S. C. Courtship song preferences in female

$721 \quad$ zebra finches are shaped by developmental auditory experience.

722 Proceedings of the Royal Society B: Biological Sciences 284, 20170054

723 (2017).

724 